\title{
Multiple scanning electrochemical microscopy mapping of tyrosinase in micro-contact printed fruit samples on polyvinylidene fluoride membrane
}

\author{
Tzu-En Lin, Fernando Cortés-Salazar, Andreas Lesch, Liang Qiao, Alexandra Bondarenko, \\ Hubert H. Girault*
}

Ecole Polytechnique Fédérale de Lausanne, Laboratoire d'Electrochimie Physique et Analytique, Station 6, CH-1015 Lausanne, Switzerland

\section{A R T I C L E I N F O}

\section{Article history:}

Received 30 January 2015

Received in revised form 30 March 2015

Accepted 31 March 2015

Available online $\mathrm{xxx}$

\section{Keywords:}

Scanning electrochemical microscopy

tyrosinase

enzymatic activity

electrochemical immunoassay readout

\begin{abstract}
A B S T R A C T
Herein, we introduce three orthogonal and compatible methods for detecting tyrosinase, a key factor in fruit browning and skin disorders, with high spatial resolution by means of scanning electrochemical microscopy (SECM). All methods are performed subsequently on the same substrate area providing a wide range of relevant information. The first SECM approach that relies on the mapping of a differential pore oxygen concentration induced by the local hydrophobic changes that the adsorption of tyrosinase generates on a porous polyvinylidene fluoride (PVDF) membrane. The second approach is based on the direct monitoring of the enzymatic activity of tyrosinase by detecting amperometrically the reaction products from the enzymatic conversion of L-3,4-dihydroxyphenylalanine (L-DOPA). Finally, tyrosinase was visualized implementing a tyrosinase sandwich immunoassay readout by SECM. The multiple SECM detection strategies were successfully applied to map unequivocally the tyrosinase enzymatic activity of a micro-contact printed banana sample. Furthermore, differential pulse voltammetry and mass spectrometry analyses were employed to elucidate the nature of the electrochemical response obtained during the tyrosinase enzymatic activity experiments.
\end{abstract}

(c) 2015 Elsevier Ltd. All rights reserved.

\section{Introduction}

Tyrosinase is an enzyme known for catalyzing the hydroxylation of monophenols and their oxidation to the respective quinones in the presence of oxygen. Furthermore, tyrosinase is directly involved in fruit ripening, in the biosynthesis of the skin pigment melanin and in skin disorders such as vitiligo (i.e. skin depigmentation due to loss of melanin) [1-6]. Therefore, the accurate and sensitive detection of tyrosinase could offer relevant information for a better understanding of different tyrosinaserelated biological processes. The latter represents a demanding task, because tyrosinase, as well as other enzymes, is normally expressed inside cells or tissues in concentration levels that are temporally and spatially dependent $[7,8]$. In proteomics, a common strategy employed to tackle such situation is based on the extraction, separation and identification of proteins by using protein electrophoresis (e.g. isoelectrofocusing (IEF) electrophoresis or sodium dodecyl sulfate polyacrylamide gel electrophoresis

\footnotetext{
* Corresponding author. Tel.: +41 02169331 51; fax: +41 0216933667 .

E-mail address: hubert.girault@epfl.ch (H.H. Girault).
}

(SDS-PAGE)), protein blotting on a suitable membrane and protein identification by using different labeling protocols [9]. Among the different employed membranes for protein blotting and detection, polyvinylidene fluoride (PVDF) is widely used since it is a highly hydrophobic and porous support with a superior protein binding capacity and a remarkable mechanical and chemical stability. Moreover, PVDF maintains the enzymatic activity of adsorbed proteins and is compatible with different protein labeling protocols including the ones based on Coomassie Blue, silver or gold staining and fluorescent or chemiluminescent dyes [10-16]. Recently, proteins immobilized on PVDF membranes have also been detected and employed for the visualization of human finger prints by scanning electrochemical microscopy (SECM) [17]. For this purpose, silver-staining [11], multi-metal-deposition (MMD) [18] or benzoquinone-tagging [19] strategies have been successfully coupled with SECM for the sensitive and selective spatial detection of immobilized proteins.

SECM is a scanning probe microscopy (SPM) technique, which is based on an ultramicroelectrode (UME) scanned in close proximity to a sample substrate and immersed in a solution containing redox active species or their precursors [20,21]. The recorded amperometric current at the UME can be spatially translated into 
local sample reactivity due to the sample capability to interact (e.g. through electron transfer reactions) with the present or generated redox species. In this way, SECM is able to image the chemical reactivity of almost any interface leading to a broad range of applications, such as the screening of electrocatalysts, the study of corrosion processes, the imaging of human fingerprints or the unraveling of different biological processes at cellular level [17,19,22-26].

Additionally, SECM enables a highly resolved spatial detection either in blotted protein separations or in the original samples based on a specific enzymatic activity or a selective tagged protein. The latter is not easily achievable by other protein detection techniques such as colorimetry, fluorescence or chemiluminescence due to additionally present interferences caused by sample color background, oxygen concentration or the presence of suspended particles in the media (i.e. scattering).

Herein we present three strategies to further extend the capabilities of SECM as a tool for the detection of adsorbed proteins on PVDF membranes. First, an indirect SECM protein detection method based on local oxygen concentration differences encountered between protein-bound and protein-free PVDF regions was implemented. Furthermore, the enzymatic activity of tyrosinase spots immobilized on PVDF membranes was studied. Finally, a third tyrosinase detection method based on the SECM readout of a tyrosinase specific immune reaction was also developed. The realized strategies were consecutively employed over the same sample region to detect spatially the presence of tyrosinase inside banana peels after microcontact printing $(\mu C P)$ on PVDF membranes. Thus, the unequivocal characterization and interpretation of the enzymatic activity of tyrosinase and other phenol oxidases on the blotted banana peels were achieved. Finally, differential pulse voltammetry (DPV) and mass spectrometry (MS) analysis were performed in order to unravel the origin of the recorded response when studying the enzymatic activity of tyrosinase by SECM.

\section{Experimental}

\subsection{Chemicals}

Tyrosinase (from mushroom, lyophilized powder, >=1000 unit/ mg solid), L-3,4-dihydroxyphenylalanine (L-DOPA, 99\%), monopotassium dihydrogen phosphate, and dipotassium monohydrogen phosphate were bought from Sigma-Aldrich (Schnelldorf, Switzerland). Sodium phosphate monobasic (99\%), sodium chloride (99.5\%), and potassium chloride ( $\geq 99.5 \%$ ) were obtained from Fluka (St. Gallen, Switzerland). Methanol ( $\geq 99 \%$ ) was purchased from Merck(Dietikon, Switzerland). PVDF membranes for protein blotting were purchased from Bio-Rad (Hercules, CA, USA). Commercial 3,3',5,5'-tetramethylbenzidine $\left(\mathrm{TMB}_{r e d}\right)$ solution was purchased from an enzyme-linked immunochemistry-based assay kit (ABRAXIS, Pennsylvania, USA). All reagents and materials were of analytical grade and used as received. Deionized water was produced by a Milli-Q plus 185 model from Millipore (Zug Switzerland).

\subsection{Immobilization of protein spots on PVDF membrane}

Squared PVDF membranes (ca. $1 \mathrm{~cm}^{2}$ ) were first wetted in methanol between 5 to 10 seconds and then transferred to water for about $1 \mathrm{~min}$. Then, the PVDF membrane was taken from the water bath and the excess of water was removed carefully with a filter paper. Afterwards, $0.8 \mu \mathrm{L}$ of a tyrosinase solution $(2 \mathrm{mg} / \mathrm{mL})$ was deposited on the membrane by using a calibrated $2.5 \mu \mathrm{L}$ micropipette (Eppendorf). To avoid the formation of any significant topographic feature, the tip of the pipette was never in physical contact with the PVDF membrane during the tyrosinase deposition process. After deposition, the immobilized tyrosinase spot on the PVDF membrane was dried under a gentle stream of nitrogen.

\subsection{SECM measurements}

SECM measurements were carried out using a home-made SECM and a typical three electrodes setup running under SECMx software (G. Wittstock, University of Oldenburg) and comprising an Ivium Compactstat (Ivium Technologies, Netherlands). A silver wire was used as the quasi-reference electrode (QRE), a platinum wire as the counter electrode (CE) and a platinum microelectrode disk ( $25 \mu \mathrm{m}$ diameter) as working electrode. All potentials given herein are referred to the QRE. Before each experiment, the platinum microelectrode was mechanically polished with a series of diamond lapping discs starting from $1 \mu \mathrm{m}$ down to $0.05 \mu \mathrm{m}$ particle sizes. The quality of the microelectrode and its $R G$ (i.e. the ratio between the radius of the insulating glass and the radius of the microelectrode $\left(r_{\mathrm{T}}\right)$ ) were determined with a Laborlux D optical microscope (Leitz, Germany). Data analyses were carried out using MIRA software [27]. The PVDF membrane was fixed on a microscope glass slide and placed on the bottom of an electrochemical setup that was completed by a Teflon body that enclosed the sample into a given volume and where the CE and QRE were placed. Prior to each SECM experiment, a leveling of the sample surface was achieved by comparing the substrate height (determined by SECM approach curves) at three corners of the square area to be scanned. A sample correction angle was applied by using a motorized tilt table (Zaber, Canada) on which the electrochemical cell was placed. All experiments were performed at room temperature $\left(20 \pm 2{ }^{\circ} \mathrm{C}\right)$.

\subsection{Microcontact printed banana on PVDF membrane}

A fresh banana cross section (ca. $0.5 \mathrm{~mm} \times 0.5 \mathrm{~mm}$ ) was cut with a scalpel blade and washed with deionized water. A PVDF membrane was prepared as mentioned above and put in contact with the banana piece for around $1 \mathrm{~min}$ (see Fig. S1 in Supporting Information(SI)). After the PVDF membrane was completely dried, a solution of $50 \mathrm{mM}$ phosphate buffer $(\mathrm{pH}=6.0)$ was employed for washing the PVDF membrane, which was then dried again and placed into the electrochemical cell for the performed experiments.

\subsection{Indirect SECM detection of adsorbed proteins on PVDF membrane by oxygen reduction method}

After the tyrosinase spot was immobilized over the PVDF membrane and placed inside the electrochemical cell, a solution of $50 \mathrm{mM}$ phosphate buffer ( $\mathrm{pH}=6.0$ ) was added into the system. Then the Pt microelectrode was biased at an electrode potential $\left(E_{\mathrm{T}}\right)$ of $-0.8 \mathrm{~V}$ to monitor the reduction of oxygen while scanning the UME closely over the PVDF sample.

\subsection{SECM detection of tyrosinase activity immobilized on PVDF membrane}

After the tyrosine spot was deposited on the PVDF membrane, a solution of $2 \mathrm{mM}$ L-DOPA in $50 \mathrm{mM}$ phosphate buffer $(\mathrm{pH}=6.0)$ was added to the system inside the electrochemical cell. Then the Pt microelectrode was scanned close to the sample and biased at $0.7 \mathrm{~V}$ to monitor the tyrosinase activity.

\subsection{SECM immunoassay of tyrosinase immobilized on PVDF membrane}

After the immobilization of a tyrosinase spot on a PVDF membrane, a solution of $1 \%$ bovine serum albumin (BSA) in $50 \mathrm{mM}$ 
phosphate buffer was employed to block the tyrosinase-free regions on the PVDF membrane. Afterwards, the BSA solution was removed and a $\sim 0.2 \mathrm{mg} / \mathrm{mL}$ solution of primary antibody was added to the system and incubated for $30 \mathrm{~min}$. Then the system was washed with PBS solution ( $\mathrm{pH}=7.4$ ). Furthermore, a $\sim 0.2 \mathrm{mg} /$ $\mathrm{mL}$ solution of secondary antibody labeled with horse radish peroxidase (HRP) was added and incubated at room temperature for another $30 \mathrm{~min}$. Finally, the system was washed 2 times with the washing solution provided by the commercial kit. The SECM read-out of the sandwich immunoassay was performed by adding a commercial solution of $\mathrm{TMB}_{\text {red }}$ and $\mathrm{H}_{2} \mathrm{O}_{2}$, which in presence of HRP produces $\mathrm{TMB}_{\text {ox }}$ that can be detected amperometrically at the scanning probe biased at $E_{\mathrm{T}}=-0.3 \mathrm{~V}$.

\section{Results and discussion}

\subsection{Indirect SECM detection of adsorbed proteins on PVDF membrane by oxygen reduction method}

The location of tyrosinase spots immobilized on a PVDF membrane was determined indirectly through a so-called oxygen reduction method [28]. Such strategy is based on the detection of oxygen at the scanning microelectrode. The concentration of oxygen in the PVDF membrane pores differs significantly between the regions with and without adsorbed proteins (Fig. 1a). Such difference results from the protein adsorption on the surface inside the pores of the PVDF membrane, which reduces locally the PVDF hydrophobicity and enables the wetting of the protein-bound PVDF pores that otherwise remain filled with air. The pore wetting is most likely caused by the polar groups of the adsorbed proteins that might be mainly exposed to the center of the pores. As a result, the concentration of oxygen in the pores filled with air (ca. $274 \mathrm{mg}$ / $\left.\mathrm{L}, 25^{\circ} \mathrm{C}, 1 \mathrm{~atm}\right)$ contrasts with the oxygen concentration in the pores filled with water ( $c a .9 \mathrm{mg} / \mathrm{L}, 25^{\circ} \mathrm{C}, 1 \mathrm{~atm}$ ). Therefore, by monitoring amperometrically the oxygen concentration at the scanning probe (by its electrochemical reduction at $E_{\mathrm{T}}=-0.8 \mathrm{~V}$ ), a clear differentiation between the zones without adsorbed proteins (depicted as higher negative current values or higher normalized currents $\left(I_{\mathrm{T}}\right)$ ) and with adsorbed proteins (depicted as lower negative current values or lower $I_{\mathrm{T}}$ ) can be achieved. Indeed, it has already been shown that SECM is a powerful tool for the spatial probing of oxygen in diffusion-limited processes as it has already been applied for the monitoring of the oxygen transport through porous materials, such as cartilages and porous membranes $[29,30]$.

The main advantage of this concept is that adsorbed protein spots on PVDF membrane can be imaged indirectly without any protein labeling procedure or by using redox mediators. Indeed, proteins are usually visualized by optical techniques based on protein staining such as silver staining [31], fluorescent dyes [15] or organic substances [32]. Although those traditional methods are powerful, they are time consuming and require the use of expensive dyes. Therefore, developing label free detection techniques of adsorbed proteins on PVDF membranes is precious.

The SECM approach curves performed on a protein-free PVDF region with a probe biased at $-0.8 \mathrm{~V}$ showed a clear increase of $I_{\mathrm{T}}$ as the concentration of $\mathrm{O}_{2}$ surrounding the air-filled PVDF pores is higher than in solution bulk (Fig. 1b). Accordingly, a current decrease is observed when the probe was brought to a working distance $(d)$ closer to the protein-bound PVDF membrane, since the concentration of $\mathrm{O}_{2}$ (that is equal inside and outside the proteinbound PVDF pores) is depleted due to its consumption at the microelectrode and the blocking of $\mathrm{O}_{2}$ diffusion towards the UME in the gap between the sample and the probe (Fig. 1b). It is expected that with such methodology a considerable current contrast between the regions with and without adsorbed proteins will be achieved, which could compete with the sensitivity of previous SECM detection techniques of adsorbed proteins (e.g. silver staining or benzoquinone tagging; see Fig. S2) $[17,19,33]$. It is important to note that the formation of reactive oxygen species (ROS) can take place during the reduction of oxygen at Pt microelectrodes, as reported elsewhere [28]. Those ROS have been demonstrated to be able to etch different substrates as elucidated by the SECM imaging of the affected areas. In the studied system, it is otherwise less likely that ROS play a major role on the observed signal since the presence of scavengers (e.g. LDOPA) in the media and the use of a highly stable substrate such as a PVDF membrane reduces certainly such possibility [34-37].

\subsection{Tyrosinase enzymatic activity detection by SECM.}

To detect the enzymatic activity of an adsorbed tyrosinase spot on PVDF membrane, a $2 \mathrm{mM}$ solution of L-DOPA (i.e. a natural tyrosinase substrate) in buffer phosphate $(50 \mathrm{mM}, \mathrm{pH}=6.5)$ was added to the system. Fig. S3a shows a cyclic voltammogram (CV) of L-DOPA where an almost steady-state signal is recorded for its (a)

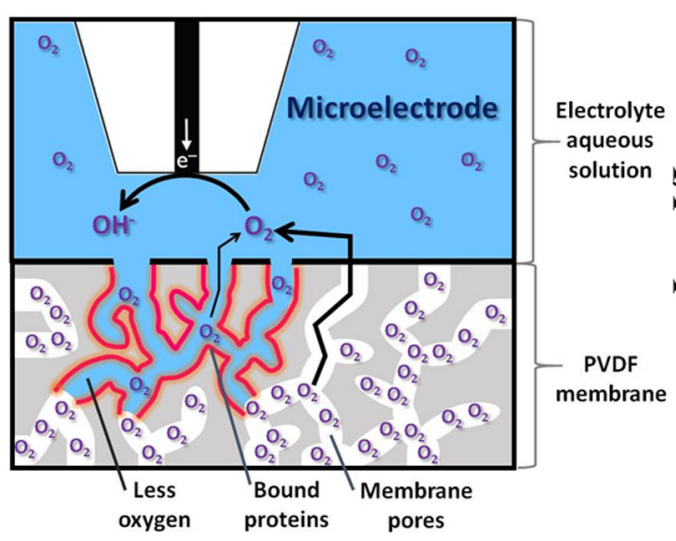

(b)

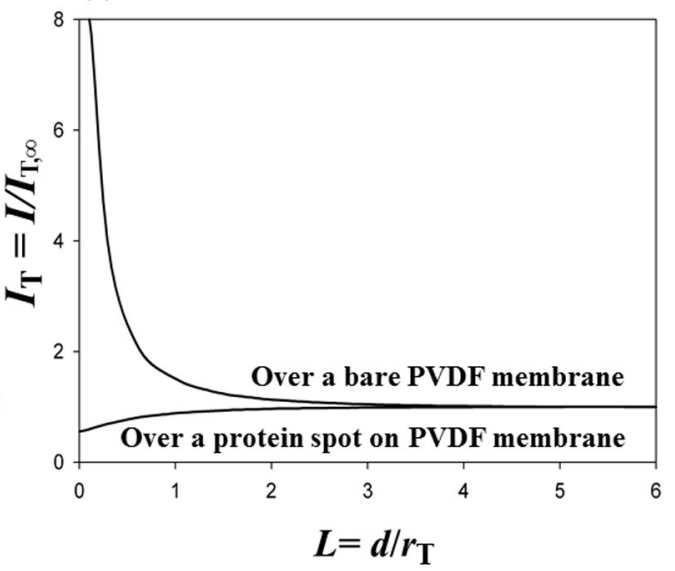

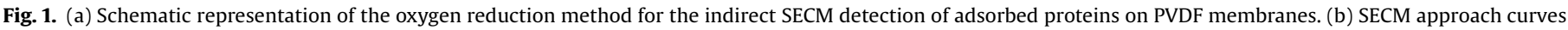

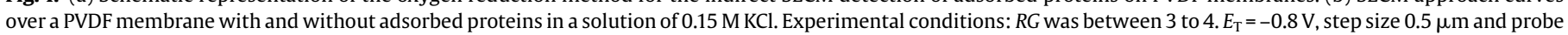
translation speed $1 \mu \mathrm{m} / \mathrm{s}$. 
electrochemical oxidation to dopaquinone. The slight tilt on the observed signal can be explained by electrode fouling taking place during the oxidation of $\mathrm{L}-\mathrm{DOPA}$ at the Pt microelectrode. Indeed, if a $\mathrm{CV}$ is taken after $2.5 \mathrm{~h}$ of applying a potential of $0.7 \mathrm{~V}$ in the same solution, a clear decrease of the current is obtained (see Fig. S4a). To avoid such passivation during the SECM experiments, an electrochemical cleaning procedure based on biasing the microelectrode at $-0.8 \mathrm{~V}$ for some seconds before applying again the oxidation potential of L-DOPA was implemented [38]. As it can be seen from chronoamperometry (CA) experiments (see Fig. S3b), the current recorded for the oxidation of L-DOPA (ca. $8 \mathrm{nA})$ is recovered almost completely after a potential step of $-0.8 \mathrm{~V}$ has been applied for $20 \mathrm{~s}$. It is important to notice that the efficient electrochemical cleaning of the electrode is achieved only at $\mathrm{pH}$ values around 6 , but not equal or higher than 7 where an even stronger electrode passivation takes place impeding any complete SECM mapping of the tyrosinase spot (results not shown). The optimum pH value for tyrosinase activity is around 7 [39], thus we expect to obtain slower kinetics in our system.

Typically, in our SECM imaging routines the microelectrode travels twice over the same line scan (i.e. forward and backward) before it is perpendicularly displaced to carry out the next line scan. In this way, two images can be recorded in one experiment by changing the conditions between forward and reverse image. Herein, during the forward line scans, a potential of $0.7 \mathrm{~V}$ for the oxidation of L-DOPA was applied, while on the reverse scans the microelectrode was switched to $-0.8 \mathrm{~V}$ for the electrochemical cleaning of the microelectrode and at the same time to detect indirectly the tyrosinase location. Before each forward line scan, a delay period was introduced to achieve the steady state conditions at the microelectrode.

The SECM image obtained in the presence of L-DOPA (Fig. 2a) depicts the tyrosinase spot as a region of higher current values. Furthermore, the application of the negative potential during the backward scan enables the indirect SECM detection of the adsorbed tyrosinase based on the oxygen detection that is in good agreement with the spatially detected tyrosinase activity, although the enzymatic activity image presents some surface heterogeneity (see Fig. 2b). The result shown in Fig. 2a is in contradiction from what has been expected, i.e. a decrease on the recorded signal over the tyrosinase active regions due to a depletion of L-DOPA within the gap between the microelectrode and sample, as the microelectrode and the enzyme compete for L-DOPA like in the SECM redox competition mode [40]. Similar results were obtained when 4-methyl catechol was employed as enzymatic substrate instead of L-DOPA (Fig. S5). The result presented in Fig. 2a suggests that in the critical time scale of the experiment an electroactive species is being generated from the enzymatic reaction between tyrosinase and L-DOPA, and that can be monitored at the scanning microelectrode biased at $0.7 \mathrm{~V}$. Indeed, in the biosynthesis pathway of melanin (Fig. 3), L-dopaquinone might lead to the formation of hydroxylated aromatic compounds that can be oxidized at similar or lower potentials than L-DOPA [41]. The underlying mechanisms of the detection of the enzymatic activity of tyrosinase in the performed SECM experiments was further investigated by using differential pulse voltammetry (DPV) and mass spectrometry (MS) experiments (vide infra).

\subsubsection{Differential pulse voltammetry (DPV) measurements}

DPV is an electrochemical technique widely used in electrochemistry for decreasing the influence of capacitive currents and for resolving different electrochemical processes that occur at similar potentials [42]. With this aim, DPV experiments in the presence of L-DOPA were performed at three different locations (see Fig. S6), i.e. in the solution bulk, over the PVDF membrane far from the tyrosinase spot and over the tyrosinase spot. An electrochemical cleaning procedure at negative potentials (i.e. $-0.8 \mathrm{~V}$ for 60 seconds) was applied between each measurement. The peak signal of highest intensity was obtained at $0.35 \mathrm{~V}$ and with two small shoulders at more positive potentials (i.e. $>0.6 \mathrm{~V}$ ) in the solution bulk (Fig. 4a). In contrast, the signal recorded $5 \mu \mathrm{m}$ above the PVDF membrane but away from the tyrosinase spot region, shows an oxidation process centered at $0.3 \mathrm{~V}$ and a lower current peak height. The decrease of the current is due to the physical blocking of the diffusion of L-DOPA towards the surface of the microelectrode at such distance. Finally, the signal recorded at $5 \mu \mathrm{m}$ over the tyrosinase spot shows a signal similar to the one

\section{(a) TYROSINASE ACTIVITY}

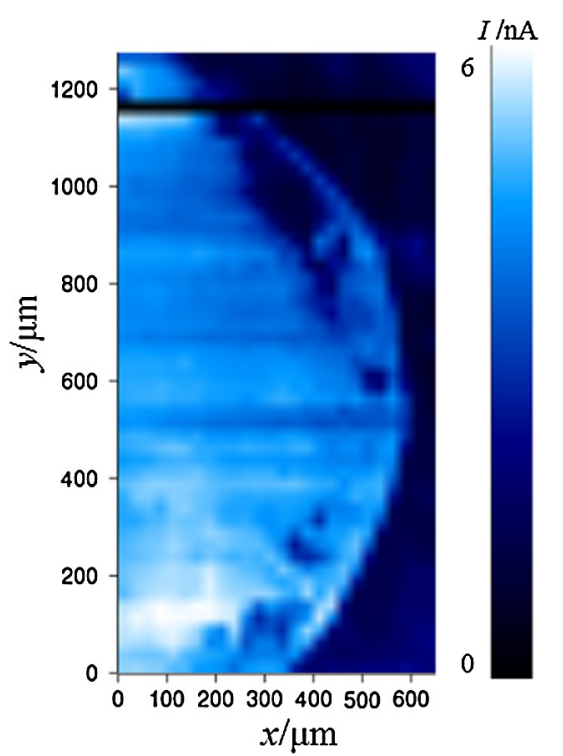

\section{(b) OXYGEN REDUCTION}

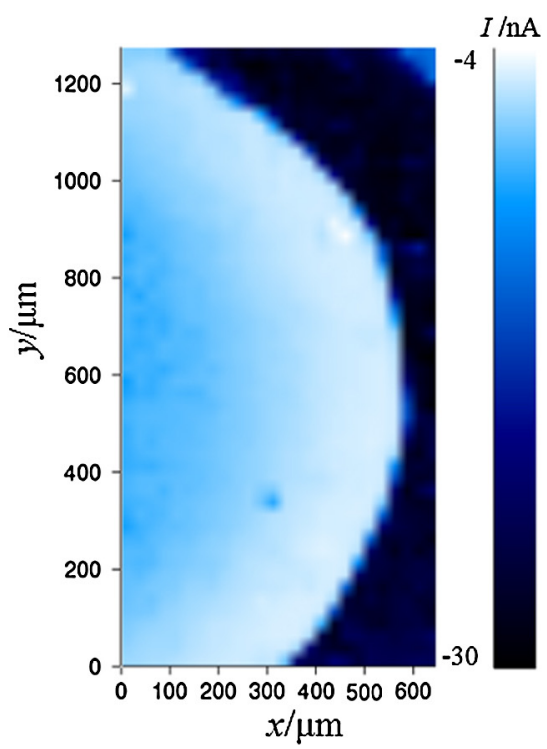

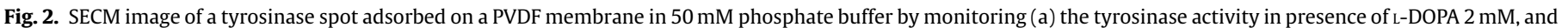

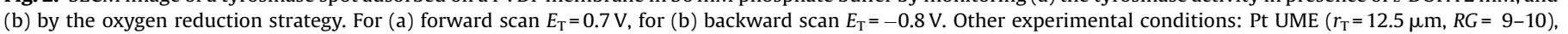
$d=7 \mu \mathrm{m}$, step size $=25 \mu \mathrm{m}$, translation speed $=20 \mu \mathrm{m} / \mathrm{s}$. 


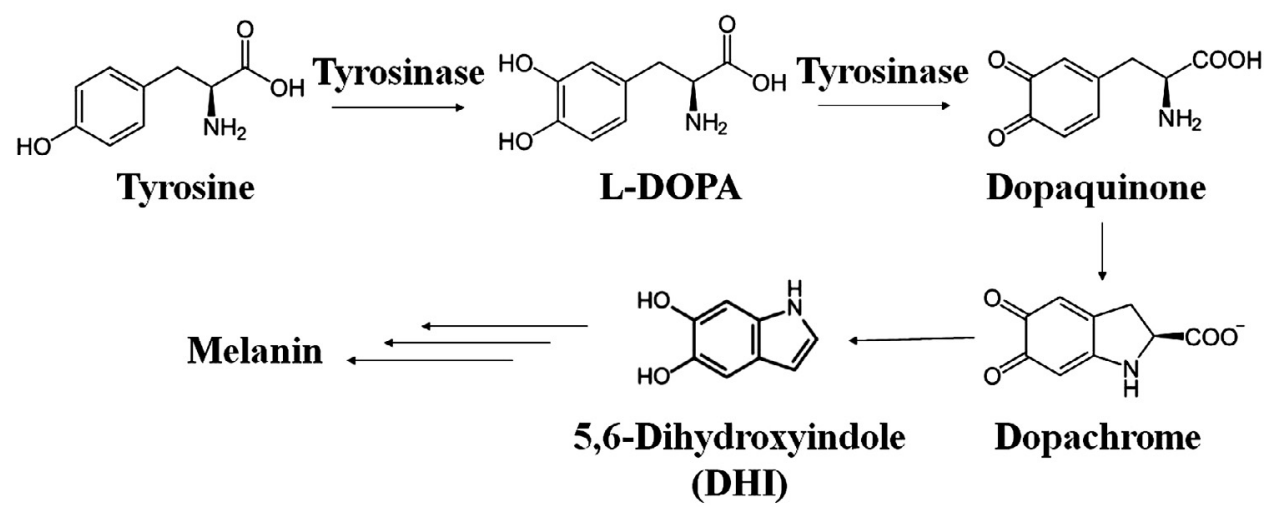

Fig. 3. Abbreviated biosynthesis pathway of melanin: starting from tyrosine and L-DOPA in the presence of tyrosinase.

(a)

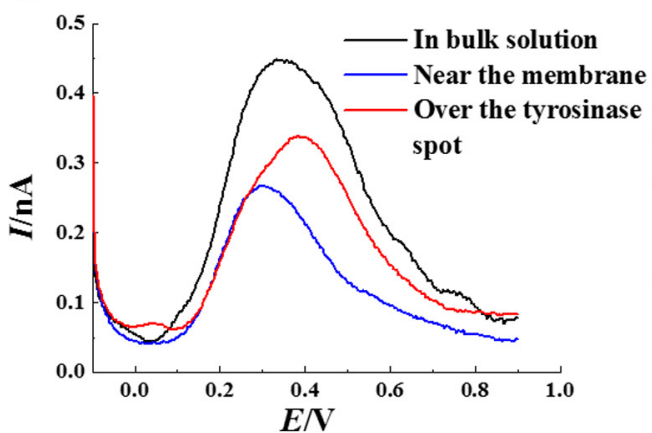

(b)

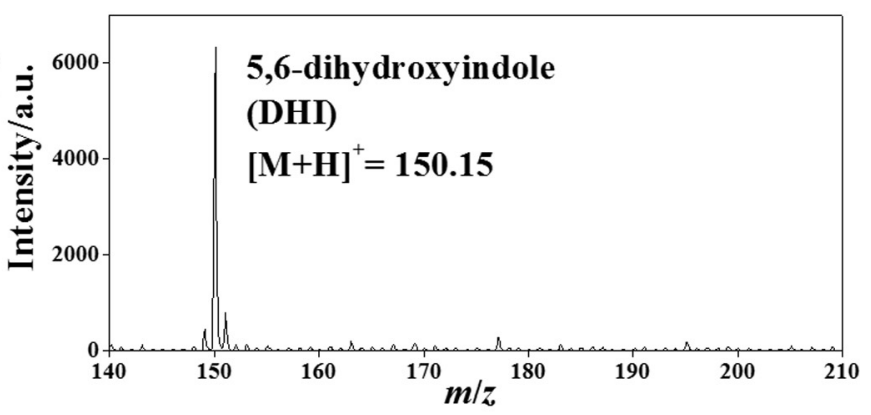

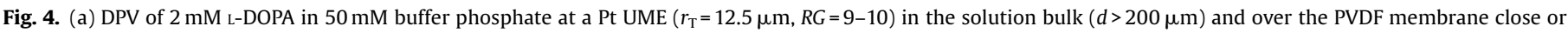

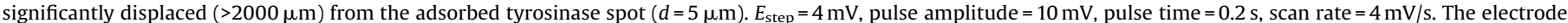

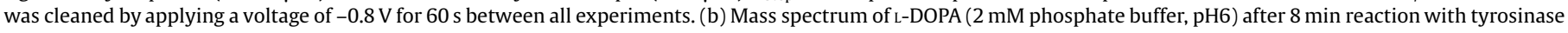
adsorbed on a PVDF membrane.

observed previously, but with a peak current height that is higher than over the PVDF region without tyrosinase. Furthermore, the observed peak shows a clear maximum at $0.4 \mathrm{~V}$, but also a shoulder at $0.3 \mathrm{~V}$ and a smaller one at $0.05 \mathrm{~V}$. These results suggest the presence of other redox species in addition to L-DOPA that might be responsible for the obtained increase in the current recorded over the tyrosinase spot. To further confirm the proposed explanation, mass spectrometry analysis was performed.

\subsubsection{Mass spectrometry analysis of the enzymatic reaction between} tyrosinase and $L-D O P A$

Fig. S7 displays the mass spectrum of a $2 \mathrm{mM}$ L-DOPA solution where a clear intense signal corresponding to the parent peak of protonated L-DOPA is observed (i.e. $[\mathrm{M}+\mathrm{H}]^{+}=198.19$ ). This result was obtained by analyzing a droplet of a $2 \mathrm{mM}$ L-DOPA solution over a tyrosinase-free PVDF membrane by using the recently developed electrostatic spray ionization (ESTASI) MS (for more details see SI-4) [43]. In contrast, when the droplet of L-DOPA solution was placed over a tyrosinase-bound PVDF region, the peak of L-DOPA disappeared rapidly after only $2 \mathrm{~min}$ due to the enzymatic reaction between L-DOPA and tyrosinase (see Fig. 4b).

Indeed, a new peak located at $150.15 \mathrm{~m} / \mathrm{z}$ appears as a result of the enzymatic reaction, which actually corresponds to 5,6-dihydroxyindole (DHI). As reported previously, DHI has been recognized as one of the products of the reaction between L-DOPA and tyrosinase [41]. CV and DPV experiments of DHI solutions showed oxidation processes taken place at potentials close to the one of L-DOPA (See Fig. S8). As a result, it can be concluded that the SECM visualization of the tyrosinase enzymatic activity is due to the detection of DHI (and perhaps other electroactive species) as the product of the enzymatic reaction between tyrosinase and L-DOPA as depicted in Fig. 5, where "Ox" indicates the oxidized product at the microelectrode.

\subsection{SECM immunoassay of tyrosinase immobilized on PVDF membrane}

The readout of immunoassays by SECM has become a powerful analytical tool for the highly sensitive and specific detection of proteins localized in a given micro-environment [44-46]. In order to verify that the detected enzymatic activity is due to the presence of tyrosinase, an immunoassay strategy has been applied to the tyrosinase spots immobilized on the PVDF membrane. With this aim, primary and HRP modified secondary antibodies were used to label the adsorbed tyrosinase, to enable its spatial detection based

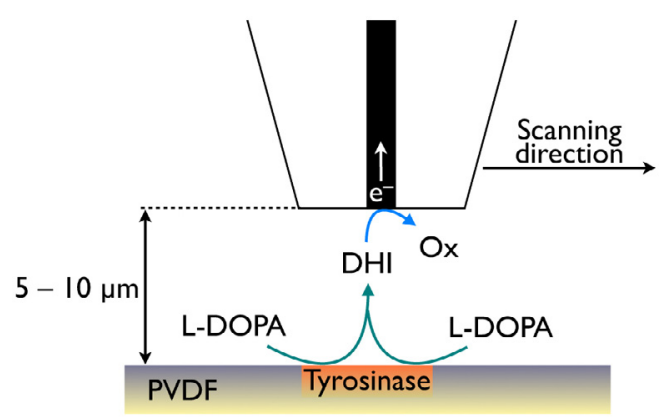

Fig. 5. Proposed principle of SECM detection of the enzymatic activity of adsorbed tyrosinase spots on PVDF membrane. 
(a)

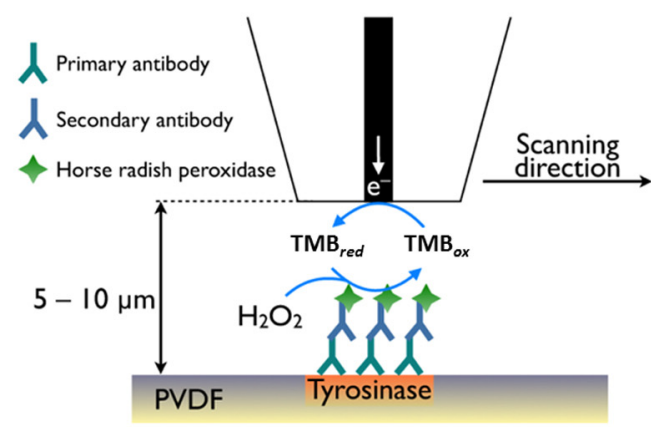

(b)

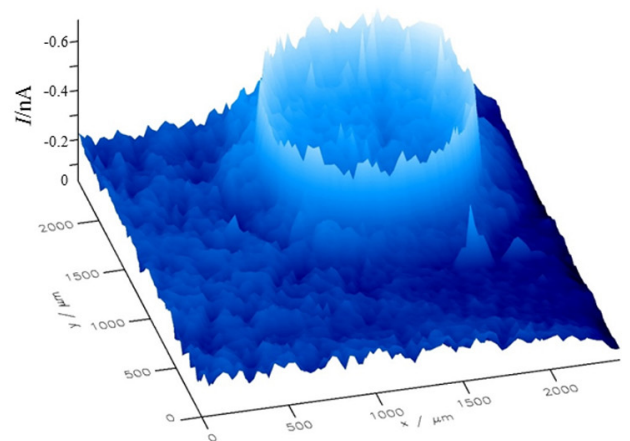

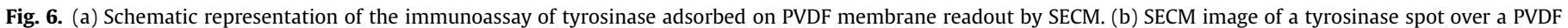

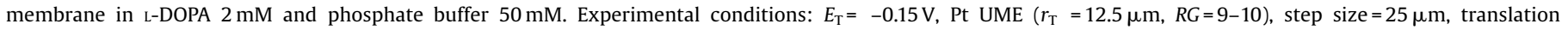
speed $=20 \mu \mathrm{m} / \mathrm{s}, d=7 \mu \mathrm{m}$.

on the amperometric monitoring of the product $\mathrm{TMB}_{\text {ox }}$ (see Fig. S9) of the enzymatic reaction between HRP and $\mathrm{TMB}_{\text {red }}$ (see Fig. 6a). In this case, the regions depicted with more negative current values correspond to the areas where tyrosinase is present (and the electrochemical reduction of $\mathrm{TMB}_{o x}$ is possible), in contrast to the less negative current regions where no tyrosinase is adsorbed (Fig. 6b). The inhomogeneous activity observed in Fig. 6b is most likely due to a coffee ring effect during the spot deposition. The main advantage of this strategy relies in the fact that tyrosinase is detected based on a specific structural recognition event. As a result, complete tyrosinase profiles in different samples can be obtained.

\subsection{SECM of $\mu C P$ banana on PVDF membrane}

For the analysis of the $\mu \mathrm{CP}$ banana sample, a tyrosinase spot was deposited next to it as a control pattern and the sample was examined in the following simultaneous order: tyrosinase activity and oxygen reduction methods (i.e. during forward and backward scans, respectively), followed by the SECM readout of the tyrosinase immunoassay (see Fig. 7). All SECM images are in good agreement with the obtained optical microscopic image of the studied sample in terms of the shape and position of the tyrosinase spot employed as control (Fig. 7a). The latter confirms the reliability of the proposed approaches. However, different features can be recognized on each one of the performed SECM experiments on the $\mu \mathrm{CP}$ banana, which is expected from such complex sample. For instance, there are areas depicting a low tyrosinase content and activity (between $x=0-500 \mu \mathrm{m}, y=0-200 \mu \mathrm{m}$, Fig. 7c and d, respectively), but that otherwise in the oxygen method image represent a protein-bound membrane region (Fig. 7b). The latter can be explained by the fact that not only adsorbed protein, but also other solid materials could be adsorbed or block the PVDF membrane pores. Still, all SECM images were able to show the absence of adsorbed protein, localization or activity of tyrosinase on the region between $x=0-500 \mu \mathrm{m}, y=400-600 \mu \mathrm{m}$ that is also in good agreement with the optical microscopic image.

It is important to notice that the distribution of tyrosinase inside the banana peel is not homogeneous and it is mainly located in certain structures of the peel. Interestingly, in Fig. 7(c) and (d), SECM images display that there is more tyrosinase in the outer part of the peel than in the inner part. Moreover, it can be concluded that in the sample analyzed, tyrosinase is the enzyme mainly involved on the oxidation of polyphenols and therefore on the

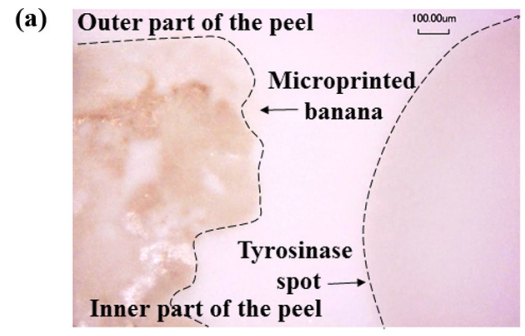

(c)

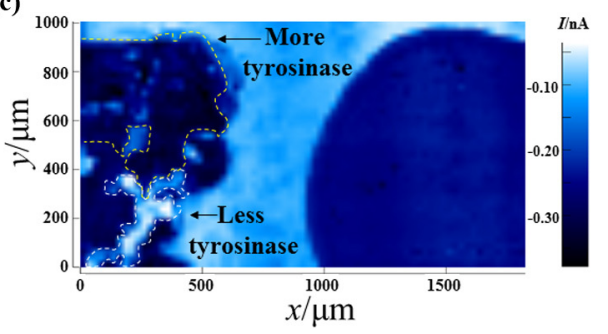

(b)

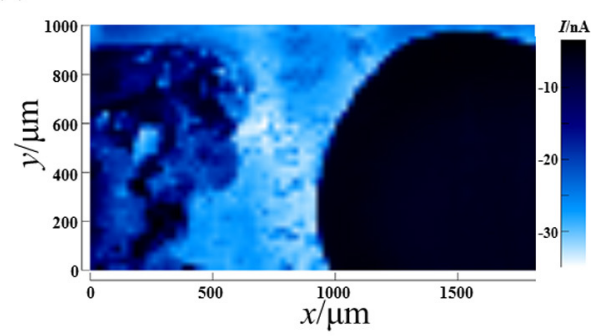

(d)

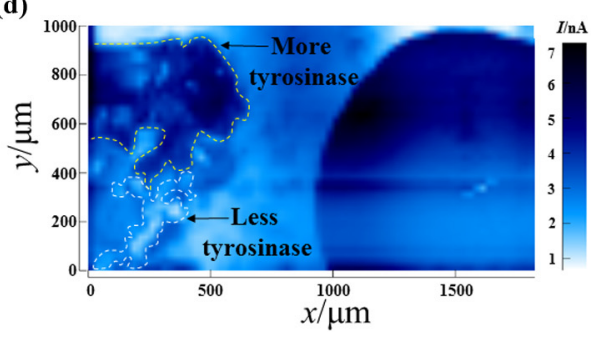

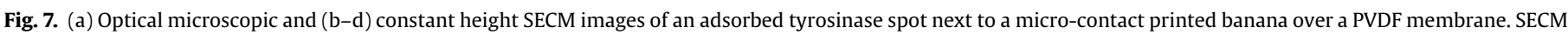

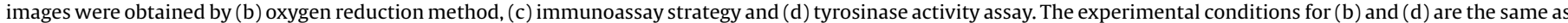

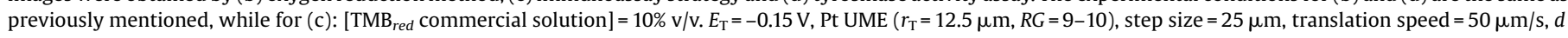
$=7 \mu \mathrm{m}$. 
banana ripening. The same strategy presented here can be apply then to study the influence of different parameters, such as temperature, oxygen concentration and presence of tyrosinase inhibitors on the kinetics of fruit ripening.

Furthermore, the clear understanding of the results obtained with such complex sample demonstrates that SECM provides all the tools required for performing a detailed characterization of biological surface processes, even by applying consecutive and compatible electrochemical strategies.

\section{Conclusions}

In the present paper, three different SECM approaches for protein detection on PVDF membranes have been implemented. First, the oxygen reduction method allows for an indirect SECM detection of adsorbed proteins based on the different oxygen concentration inside the PVDF membrane pores, as a consequence of the induced local changes on the membrane hydrophobicity. Furthermore, the detection of tyrosinase enzymatic activity was also developed and its principle of detection was examined and confirmed through DPV and MS analysis. Finally, the specific recognition of tyrosinase spots was possible via the SECM readout of an immunoassay strategy. Thus, tyrosinase spots and $\mu \mathrm{CP}$ banana deposited on PVDF membranes were consecutively studied using the three proposed SECM strategies. As a result, a clear and straightforward interpretation of the localization and activity of tyrosinase inside the banana peels was achieved. Besides banana, other biological samples that contain proteins can be $\mu C P$ on PVDF membranes and similarly analyzed by SECM. It can be envisaged that SECM studies of immobilized tyrosinase on PVDF membrane can be used to understand the influence of different factors on the binding process between tyrosinase and autoimmune antibodies typically generated in Vitiligo patients and which are known to be the responsible for the propagation of this disease. Thus, SECM can be employed as a powerful tool for disease diagnostics and molecular screening through electrochemical readout.

\section{Acknowledgements}

Tzu-En Lin gratefully acknowledges Taiwan government for “The 2013 MOE Technologies Incubation Scholarship".

\section{Appendix A. Supplementary data}

Supplementary data associated with this article can be found, in
the online version, at http://dx.doi.org/10.1016/j.
electacta.2015.03.224.

\section{References}

[1] M. Joslyn, J. Ponting, Enzyme-catalyzed oxidative browning of fruit products Adv. Food Res. 3 (1951) 1-44.

[2] Y. Jiang, X. Duan, D. Joyce, Z. Zhang, J. Li, Advances in understanding of enzymatic browning in harvested litchi fruit, Food Chem. 88 (2004) 443-446.

[3] V. Kanh, Effect of Proteins, Protein Hydrolyzates and Amino Acids on o-Dihydroxyphenolase Activity of Polyphenol Oxidase of Mushroom, Avocado, and Banana, J. Food Sci. 50 (1985) 111-115.

[4] K.C. Hertz, L.A. Gazze, C.H. Kirkpatrick, S.I. Katz, Autoimmune vitiligo: detection of antibodies to melanin-producing cells, N. Engl. J. Med. 297 (1977) 634-637.

[5] C. Betterle, A. Peserico, G. Bersani, Vitiligo and autoimmune polyendocrine deficiencies with autoantibodies to melanin-producing cells, Arch Dermatol 115 (1979) 364

[6] Y. Song, E. Connor, Y. Li, B. Zorovich, P. Balducci, N. Maclaren, The role of tyrosinase in autoimmune vitiligo, The Lancet 344 (1994) 1049-1052.

[7] W.F. Patton, Detection technologies in proteome analysis, J. Chromatogr. B 771 (2002) 3-31.
[8] S. Sriyam, S. Sinchaikul, P. Tantipaiboonwong, C. Tzao, S. Phutrakul, S.T. Chen, Enhanced detectability in proteome studies, J. Chromatogr. B 849 (2007) 91-104.

[9] H.J. Issaq, The role of separation science in proteomics research, Electrophoresis 22 (2001) 3629-3638.

[10] K. Berggren, T.H. Steinberg, W.M. Lauber, J.A. Carroll, M.F. Lopez, E. Chernokalskaya, L. Zieske, Z. Diwu, R.P. Haugland, W.F. Patton, A luminescent ruthenium complex for ultrasensitive detection of proteins immobilized on membrane supports, Anal. Biochem. 276 (1999) 129-143.

[11] T. Rabilloud, L. Vuillard, C. Gilly, J.J. Lawrence, Silver-staining of proteins in polyacrylamide gels: a general overview, Cell. Mol. Biol. 40 (1994) 57-75.

[12] V. Neuhoff, N. Arold, D. Taube, W. Ehrhardt, Improved staining of proteins in polyacrylamide gels including isoelectric focusing gels with clear background at nanogram sensitivity using Coomassie Brilliant Blue G-250 and R-250, Electrophoresis 9 (1988) 255-262.

[13] T.H. Steinberg, B.J. Agnew, K.R. Gee, W.Y. Leung, T. Goodman, B. Schulenberg, J. Hendrickson, J.M. Beechem, R.P. Haugland, W.F. Patton, Global quantitative phosphoprotein analysis using multiplexed proteomics technology, Proteomics 3 (2003) 1128-1144.

[14] C.R. Merril, Gel-staining techniques, Methods Enzymol. 182 (1990) 477-488.

[15] A. Mayer, S. Neuenhofer, Luminescent labels - More than just an alternative to radioisotopes? Angew. Chem. Int. Ed. 33 (1994) 1044-1072.

[16] R. Westermeier, R. Marouga, Protein detection methods in proteomics research, Biosci. Rep. 25 (2005) 19-32.

[17] M. Zhang, H.H. Girault, SECM for imaging and detection of latent fingerprints, Analyst 134 (2009) 25-30.

[18] M. Zhang, A. Becue, M. Prudent, C. Champod, H.H. Girault, SECM imaging of MMD-enhanced latent fingermarks, Chem. Commun. (2007) 3948-3950.

[19] F. Cortés-Salazar, J.-M. Busnel, F. Li, H.H. Girault, Adsorbed protein detection by scanning electrochemical microscopy, J. Electroanal. Chem. 635 (2009) 69-74.

[20] P.R. Unwin, A.J. Bard, Scanning electrochemical microscopy. 9. Theory and application of the feedback mode to the measurement of following chemical reaction rates in electrode processes, J. Phys. Chem. 95 (1991) 7814-7824.

[21] S. Amemiya, A.J. Bard, F.-R.F. Fan, M.V. Mirkin, P.R. Unwin, Scanning electrochemical microscopy, Annu. Rev. Anal. Chem. 1 (2008) 95-131.

[22] F. Zhang, V. Roznyatovskiy, F.-R.F. Fan, V. Lynch, J.L. Sessler, A.J. Bard, A Method for Rapid Screening of Photosensitizers by Scanning Electrochemical Microscopy (SECM) and the Synthesis and Testing of a Porphyrin Sensitizer, J. Phys. Chem. C 115 (2011) 2592-2599.

[23] H. Ye, J. Lee, J.S. Jang, A.J. Bard, Rapid screening of BiVO4-based photocatalysts by scanning electrochemical microscopy (SECM) and studies of their photoelectrochemical properties, J. Phys. Chem. C 114 (2010) 13322-13328.

[24] R.M. Souto, Y. González-García, D. Battistel, S. Daniele, On the use of mercurycoated tips in scanning electrochemical microscopy to investigate galvanic corrosion processes involving zinc and iron, Corros. Sci. 55 (2012) 401-406.

[25] M. Pähler, J.J. Santana, W. Schuhmann, R.M. Souto, Application of AC-SECM in Corrosion Science: Local Visualisation of Inhibitor Films on Active Metals for Corrosion Protection, Chem. -Eur. J. 17 (2011) 905-911.

[26] L.P. Bauermann, W. Schuhmann, A. Schulte, An advanced biological scanning electrochemical microscope (Bio-SECM) for studying individual living cells, Phys. Chem. Chem. Phys. 6 (2004) 4003-4008.

[27] G. Wittstock, T. Asmus, T. Wilhelm, Investigation of ion-bombarded conducting polymer films by scanning electrochemical microscopy (SECM), Fresenius. J. Anal. Chem. 367 (2000) 346-351.

[28] J.-M. Noël, A. Latus, C. Lagrost, E. Volanschi, P. Hapiot, Evidence for OH radical production during electrocatalysis of oxygen reduction on Pt surfaces: consequences and application, J. Am. Chem. Soc. 134 (2012) 2835-2841.

[29] B.D. Bath, R.D. Lee, H.S. White, E.R. Scott, Imaging molecular transport in porous membranes. Observation and analysis of electroosmotic flow in individual pores using the scanning electrochemical microscope, Anal. Chem. 70 (1998) 1047-1058.

[30] M. Gonsalves, A.L. Barker, J.V. Macpherson, P.R. Unwin, D. O'Hare, C.P. Winlove, Scanning electrochemical microscopy as a local probe of oxygen permeability in cartilage, Biophys. J. 78 (2000) 1578-1588.

[31] T. Rabilloud, L. Vuillard, C. Gilly, J. Lawrence, Silver-staining of proteins in polyacrylamide gels: a general overview, Cell. Mol. Biol. 40 (1994) 57.

[32] V. Neuhoff, N. Arold, D. Taube, W. Ehrhardt, Improved staining of proteins in polyacrylamide gels including isoelectric focusing gels with clear background at nanogram sensitivity using Coomassie Brilliant Blue G-250 and R-250, Electrophoresis 9 (1988) 255-262.

[33] F. Cortés-Salazar, M. Zhang, A. Becue, J.M. Busnel, M. Prudent, C. Champood, H. H. Girault, Human fingerprint imaging by Scanning Electro-Chemical Microscopy (SECM), Chimia 63 (2009) 580.

[34] D. Wang, K. Li, W. Teo, Preparation and characterization of polyvinylidene fluoride (PVDF) hollow fiber membranes, J. Membr. Sci. 163 (1999) $211-220$.

[35] D. Wang, K. Li, W. Teo, Porous PVDF asymmetric hollow fiber membranes prepared with the use of small molecular additives, J. Membr. Sci. 178 (2000) $13-23$.

[36] J. Ji, F. Liu, N.A. Hashim, M.M. Abed, K. Li, Poly (vinylidene fluoride)(PVDF) membranes for fluid separation, Reactive and Functional Polymers 86 (2015) 134-153.

[37] S. Lewis, V. Smuleac, A. Montague, L. Bachas, D. Bhattacharyya, Ironfunctionalized membranes for nanoparticle synthesis and reactions, Sep. Sci. Technol. 44 (2009) 3289-3311. 
[38] J.-M. Noël, Y. Yu, M.V. Mirkin, Dissolution of Pt at moderately negative potentials during oxygen reduction in water and organic media, Langmuir 29 (2013) 1346-1350.

[39] H. Saeki, A. Oikawa, Effects of $\mathrm{pH}$ and type of sugar in the medium on tyrosinase activity in cultured melanoma cells, J. Cell. Physiol. 94 (1978) 139-145.

[40] A. Dobrzeniecka, A. Zeradjanin, J. Masa, J. Stroka, M. Goral, W. Schuhmann, P.J. Kulesza, Scanning Electrochemical Microscopy for Investigation of Multicomponent Bioelectrocatalytic Films, ECS Trans. 35 (2011) 33-44.

[41] S. Gidanian, P.J. Farmer, Redox behavior of melanins: direct electrochemistry of dihydroxyindole-melanin and its $\mathrm{Cu}$ and $\mathrm{Zn}$ adducts, J. Inorg. Biochem. 89 (2002) 54-60.

[42] A.J. Bard, F. Corte's-Salazar, H.H. Girault, Additional Recent Applications and Prospects, in: A.J. Bard, M.V. Mirkin (Eds.), Scanning electrochemical microscopy, CRC Press, 2012, pp. 635-646.
[43] L. Oiao, R. Sartor, N. Gasilova, Y. Lu, E. Tobolkina, B. Liu, H.H. Girault Electrostatic-spray ionization mass spectrometry, Anal. Chem. 84 (2012) $7422-7430$.

[44] W. Song, Z. Yan, K. Hu, Electrochemical immunoassay for CD10 antigen using scanning electrochemical microscopy, Biosens. Bioelectron. 38 (2012) 425-429.

[45] W.S. Roberts, F. Davis, J.L. Holmes, S.D. Collyer, L.D. Larcombe, S.L. Morgan, S.P. Higson, Detection and imaging the expression of the trans-membrane protein CD44 in RT112 cells by use of enzyme-labeled antibodies and SECM, Biosens. Bioelectron. 41 (2013) 282-288.

[46] H. Shiku, Y. Hara, T. Matsue, I. Uchida, T. Yamauchi, Dual immunoassay of human chorionic gonadotropin and human placental lactogen at a miocrofabricated substrate by scanning electrochemical microscopy, J. Electroanal. Chem. 438 (1997) 187-190. 